\title{
COGNITIVE AND PSYCHOMOTOR CORRELATES OF SELF-REPORTED DRIVING SKILLS AND BEHAVIOR
}

\author{
Nebi Sümer ${ }^{1}$, H. Belgin Ayvasik ${ }^{1}$, Nurhan Er $^{2}$ \\ ${ }^{1}$ Middle East Technical University, Ankara, Turkey \\ ${ }^{2}$ Ankara University, Ankara, Turkey \\ E-mail: nsumer@metu.edu.tr
}

\begin{abstract}
Summary: The purpose of this study is to predict self-reported driving and safety skills, traffic violations, and errors by using the measures of cognitive and psychomotor abilities. Male drivers $(\mathrm{N}=716$, mean age $=36.59)$ were administered the computerized measures of monotonous and selective attention, visual pursuit, eye-hand coordination, reaction time, and peripheral perception. They also responded to the measures of driving skills and behaviors. Examination of the correlations indicated that the indices of visual pursuit, coordination, peripheral perception, and reaction time significantly correlated with driving skills and aberrant behaviors. The results of the sequential regression analyses controlling for age, level of education, and annual $\mathrm{km}$ revealed that selective attention negatively and significantly predicted both types of skills and positively predicted violations and errors. Peripheral perception, visual pursuit, and reaction time were the significant predictors of driving skills and errors in the expected direction. Cognitive and psychomotor abilities accounted for $11 \%$ to $17 \%$ of the variances in the self-reported driving variables. Results suggested that although the magnitude of the associations was relatively weak, psychomotor and cognitive/perceptual abilities are associated with self-reported driving performance and behaviors for young and middle-aged drivers. These findings indicated that certain measures of cognitive and psychomotor abilities, such as peripheral perception and selective attention, have implications for driver assessment systems and should be carefully examined in future research.
\end{abstract}

\section{INTRODUCTION}

Epidemiological statistics in Turkey show that traffic accidents are prominent causes of premature mortality and death, especially among young male drivers (SWEROAD Report, 1999). In recent years, a number of regulatory legislations for preventive purposes have been initiated in Turkey. One of these legislations accepted in 1997 mandates drivers who have a certain number of driving convictions to take computerized cognitive and psychomotor tests for screening purposes. We developed a computerized psycho-technical assessment system including a number of tests measuring cognitive and psychomotor performance (Sumer, Ayvasik, \& Er, 2002). This study aims to examine cognitive and psychomotor correlates of self-reported driving skills and behaviors by using a subset of the tests in this system.

\section{Driving Performance and Cognitive-Psychomotor Abilities}

Previous studies have shown that both young and older drivers have higher risk of traffic crashes than other age groups and they differ in their cognitive and psychomotor abilities. It is well documented that the primary problems with young drivers are risk taking and lack of skills and 
the primary problems with old drivers are mental and physical deficits (e.g., McGwin \& Brown, 1999; Evans, 1991). Hence, the role of cognitive, perceptual, and psychomotor abilities in driving performance was dominantly investigated among older drivers (e.g., HakamiesBlomqvist, 1998). McKnight and McKnight (1999) classified abilities related to driving performance into five domains: (1) sensory (e.g., visual acuity and contrast sensitivity), (2) attentional (e.g., general and selective attention), (3) perceptual (e.g., speed of perception and motion detection, (4) cognitive (e.g., short term memory), and (5) psychomotor (e.g., reaction time, coordination, and tracking). Although most of these domains, especially higher order cognitive skills such as hazard perception, have been examined in relation to driving performance and accident involvement (see Lawton and Parker, 1998, for a review), they have not been satisfactorily examined in relation to the safety performance and aberrant driver behaviors of young and middle age drivers.

\section{Self-Reported Driving Skills and Behaviors}

Elander, West, and French (1993) made a distinction between driving behavior (i.e., style) and driving skills (i.e., perceptual-motor performance) and asserted that driver behavior is associated with personality characteristics, attitudes, beliefs, needs, and values. They can also be a proximal factor for accident involvement (Sümer, 2003). Recent studies have shown that driver behaviors measured by self-reported measures, such as the Driver Behavior Questionnaire (DBQ; Reason et al., 1990) predict accident involvement and risky driving (e.g., Parker \& Stradling, 2001). Aberrant driving behaviors, especially traffic violations and errors, appeared to be the serious risk factors (Sümer, 2003). Driving skills, however, reflect the person's actual performance on the road and they can be improved by practice and training. Lajunen and Summala (1995) asserted that safety related skills (defined as the anticipatory accident avoidance skill) should also be included in the assessment of perceptual-motor skills. These authors suggested that the distinction between driving and safety skills is critical because the driver's internal balance between these skills reflects the driver's attitude to safety. Thus, Lajunen and Summala (1995) developed the Driver Skill Inventory (DSI) to assess both general perceptual-motor performance and safety concerns. Although both driver behaviors and skills have been examined in relation to accident risk, their links with the cognitive, perceptual, and psychomotor abilities have not been studied.

Recently, driver performance and aberrant driver behaviors have been commonly investigated by using self-report measures and these measures were shown to have satisfactory validity in predicting actual driving behaviors on the road and accident involvement (Elander et al., 1993). In this study, we assumed that predictive power of cognitive and psychomotor abilities on driving skills and behaviors would be examined using self-report measures. We aimed to employ specific measures representing the main domains of cognitive and psychomotor performance related to driving.

Furthermore, past studies demonstrated that both young (Brown \& Groeger, 1988) and older drivers (Marottoli \& Richardson, 1998) tend to overestimate their driving skills in self-reported measures. Although self-reported measures of driving skills and behaviors are biased and seem to reflect some sort of overconfidence effect, we still expected that drivers' level of cognitive and psychomotor abilities would be systematically associated with their driving skills and behaviors. Thus, specifically, we expected that driving skills (rather than safety skills) and errors rather than violations) would be relatively strongly predicted by cognitive and psychomotor abilities. 


\section{METHODS}

\section{Participants and Procedure}

Both amateur and professional drivers $(\mathrm{N}=716)$ voluntarily participated in the study. The majority of the participants were young and middle aged drivers (mean age $=36.59, \mathrm{SD}=7.60$, range $=20$ - 61). Of the participants, $31 \%$ were elementary school, $47 \%$ were middle and high school, and $22 \%$ were university graduates. Following the completion of practice trials in each test, drivers were administered computer-based cognitive and psychomotor tests in a research center established to investigate the validity of a psycho-technical driver assessment system (see Figure 1). Following the completion of computerized tests, participants filled out the scales for driving skills and behaviors and a demographic measure. Completion of all tests took 90 to 120 minutes, depending on the drivers' level of education.

\section{Instruments}

Instruments consisted of a questionnaire and the computerized cognitive and psychomotor test battery explained below. Computerized tests were developed by the researchers for a project aiming to assess cognitive and psychomotor skills of the convicted drivers in Turkey.

Traffic Monotonous Attention Test (TMAT). The TMAT is a computer-based cancellation task consisting of differently designed versions of a road traffic sign arranged in an 11X17 matrix. The task was to identify 50 items identical to the target item (out of 186 items) within the matrix. The participants were given a maximum of 2.5 minutes to complete the task. The number of correct (hits), incorrect (commissions), and missing (omissions) responses and the time taken to complete the task were recorded for each participant. An index was calculated representing the ratio of the correct responses to the incorrect and omitted ones and it was used in the analyses.

Selective Attention Test (SAT). The SAT consists of 60 items each having six traffic signs placed in a 4X4 matrix of squares. Traffic signs were filled in the last three squares of the first row and column. The rest of the squares were unfilled. One of the traffic signs on the row and column was identical to each other and the drivers were asked to find the identical signs and touch the crossing point (square) of these signs on the matrix. Each matrix was presented for $3 \mathrm{sec}$. As in the TMAT, the number of correct (hits), incorrect (commissions), and missing (omissions) responses and the time taken to complete the task were recorded for each participant.

Visual Pursuit Test (VPT). VPT is a type of visual attention test aiming to assess drivers' visual plasticity and tracking ability in a complex labyrinth task. In the VPT, there were 10 complicated lines placed in a circle each starting from a different point on the edge of the circle. Drivers were asked to find the end points of the randomly presented lines by tracking with their eyes without using their hands. The number of the correct responses was calculated representing drivers' performance on the test.

Eye-Hand Coordination Test (CT). CT aims to assess drivers' motor coordination skill between the eyes and hands. In both routine and complex road trails, which were simulated on the screen, drivers were asked to drive the car by trying to stay in the designated lane. Speed of the car was controlled by the computer and both speed of the car and the complexity of the road were 
manipulated. The duration (seconds) drivers stayed out of the designated road and lanes were calculated to depict the coordination ability. Therefore, higher scores represented lower levels of coordination skill.

Reaction Time (RT) Test. RT was measured by employing a series of compatible and controlled reactions to the given stimuli. Drivers were instructed to react by pressing a button as soon as they saw a green square or circle on the screen and not to respond when they saw red ones. When they saw the circles, they were asked to press with their hands and when they saw squares, they were asked to press with their feet. Stimuli on the right and left side were responded with the corresponding hand or foot. Only reactions given with feet were calculated in this study.

Peripheral Perception under Distracted Attention Condition. Assesses drivers' ability to pay attention and detect the stimuli coming from peripheral panels extending from the monitor with $140^{\circ}$ angle while simultaneously attending to a demanding task (i.e., a color-matching task) on the screen (refer to Figure 1 for the system). Drivers were asked to detect randomly delivered lights from left and right panels by pressing a pedal with their matching foot.

Driver Behaviors Questionnaire (DBQ). The Turkish version of the Driver Behavior Questionnaire (DBQ; Reason, Manstead, Stradling, Baxter, \& Campbell, 1990) with additional items was used to measure driver behaviors. (e.g., disregard for the speed limit on a residential road). The DBQ was used in previous studies in Turkey on similar samples (e.g., Sümer, 2003). Participants were asked to indicate how often they committed each of the behaviors in the previous year on a six-point scale $(0=$ never, $5=$ nearly all the time). Factor analyses on the Turkish DBQ items yielded two interpretable factors representing driving violations and inattention errors. Reliability coefficients were satisfactory for both subscales $(\alpha=.89$ and .79 , respectively).

Driver Skill Inventory (DSI). The DSI is a 20-item self-reported measure of perceptual motor and safety skills developed by Lajunen and Summala (1995). The DSI was translated into Turkish and shown to have high reliability and validity coefficients. Drivers were asked to rate how weak or strong they were on the given skills by using 5 -point scales $(0=$ very weak and $4=$ very strong). The DSI has two subscales, driving (perceptual-motor) skills (e.g., fluent driving) and safety skills (e.g., "conforming to the speed limits"). Reliability coefficients were satisfactory for both subscales ( $\alpha=.89$ and .83 , respectively).

\section{RESULTS}

Correlations between the computerized tests and self-reported driving variables are presented in Table 1. As expected, as age increases both cognitive and psychomotor performance deteriorate. Although the majority of the correlations between computerized tests and driver skills and behaviors were significant, they were weak to moderate in size. The driving skills and behaviors were moderately strongly correlated with peripheral perception and reaction time. Those with higher levels of peripheral perception ability and quicker reaction time tended to report higher levels of driving and safety skills and lower levels of violations and error.

A series of sequential regression analyses was conduced to predict the outcome variables after controlling for the demographic characteristics. As seen in Table 2, level of education was 


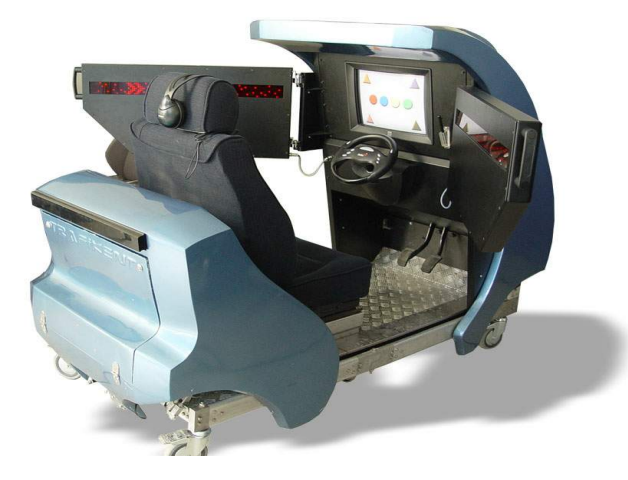

Figure 1. Trafikent Psycho-Technical Driver Assessment System for computerized measures of cognitive and psychomotor abilities.

Table 1. Descriptive statistics and correlations among the major variables of the study

\begin{tabular}{|c|c|c|c|c|c|c|c|c|c|c|c|c|c|}
\hline Variables & 1 & 2 & 3 & 4 & 5 & 6 & 7 & 8 & 9 & 10 & 11 & 12 & 13 \\
\hline \multicolumn{14}{|l|}{ Demographics } \\
\hline 1. Age & 1.00 & & & & & & & & & & & & \\
\hline 2. Education ${ }^{1}$ & $-.11 * *$ & 1.00 & & & & & & & & & & & \\
\hline 3. Exposure (annual km) & $.08^{*}$ & .05 & 1.00 & & & & & & & & & & \\
\hline \multicolumn{14}{|l|}{ Self-report measures } \\
\hline 4. Driving Skills & -.05 & $.44 * * *$ & $.14 * * *$ & 1.00 & & & & & & & & & \\
\hline 5. Safety Skills & -.01 & $.43 * * *$ & .04 & $.66^{* * *}$ & 1.00 & & & & & & & & \\
\hline 6. Violations & -.03 & $-.33 * * *$ & -.01 & $-.46 * * *$ & $-.68 * * *$ & 1.00 & & & & & & & \\
\hline 7. Inattention error & .05 & $-.48 * * *$ & .01 & $-.56 * * *$ & $-.52 * * *$ & $.68 * * *$ & 1.00 & & & & & & \\
\hline \multicolumn{14}{|l|}{ Computer-based measures } \\
\hline 8. Monotonous attention & $-.17 * * *$ & $.13 * * *$ & -.04 & -.05 & $-.10^{*}$ & .06 & .03 & 1.00 & & & & & \\
\hline 9. Selective attention & $-.31 * * *$ & $.08 *$ & -.04 & $-.16^{* * *}$ & $-.26 * * *$ & $.22 * * *$ & $.09 *$ & $.41 * * *$ & 1.00 & & & & \\
\hline 10. Visual pursuit & $-.27 * * *$ & $.30 * * *$ & -.05 & $.16^{* * *}$ & $.15 * * *$ & $-.22 * * *$ & $-.31 * * *$ & $.26 * * *$ & $.36 * * *$ & 1.00 & & & \\
\hline 11. Eye-hand coordination & $.15 * * *$ & $-.21 * * *$ & .04 & $-.13 * * *$ & $-.10^{*}$ & $.13 * * *$ & $.10 *$ & $-.17 * * *$ & $-.22 * * *$ & $-.19 * * *$ & 1.00 & & \\
\hline 12. Reaction time & $.16^{* * *}$ & $-.42 * * *$ & .03 & $-.35 * * *$ & $-.32 * * *$ & $.30 * * *$ & $.43 * * *$ & .02 & .01 & $-.31 * * *$ & $.21 * * *$ & 1.00 & \\
\hline 13. Peripheral perception & $-.26 * * *$ & $.53 * * *$ & .02 & $.43 * * *$ & $.44 * * *$ & $-.35 * * *$ & $-.47 * * *$ & -.06 & .03 & $.37 * * *$ & $-.14 * * *$ & $-.54 * * *$ & 1.00 \\
\hline Means & 36.59 & 1.90 & 22758 & 34.92 & 22.86 & 18.95 & 11.26 & 67.12 & 53.18 & 67.93 & 9623.22 & 1532.87 & 34.48 \\
\hline Standard Deviations & 7.59 & .72 & 20714 & 5.89 & 4.79 & 12.90 & 7.96 & 18.73 & 17.45 & 18.64 & 9871.45 & 245.44 & 13.57 \\
\hline
\end{tabular}

$* \mathrm{p}<.05, * * \mathrm{p}<.01, * * * \mathrm{p}<.001 .{ }^{1}$ Level of education was measured as $1=$ elementary school, $2=$ secondary and high school, $3=$ university.

Note: Higher scores on monotonous attention, selective attention, visual pursuit, and peripheral perception, represent higher levels of these abilities. Higher scores on eye-hand coordination and reaction time represent lower levels of these abilities. 
Table 2. Sequential regression analyses on the outcome variables

\begin{tabular}{lllll}
\hline & Driving Skills $(\beta)$ & Safety Skills $(\beta)$ & Violations $(\beta)$ & Errors $(\beta)$ \\
\hline Step 1 & & & & \\
Age & -.04 & .03 & -.05 & .02 \\
Education & $.44^{* * *}$ & $.40^{* * *}$ & $-.32^{* * *}$ & $-.50^{* * *}$ \\
$\quad$ Exposure (annual km) & $.10^{* *}$ & .01 & .04 & .05 \\
$\mathrm{R}^{2}$ Change & .21 & .16 & .10 & .25 \\
Step 2 & & & & \\
Age & -.03 & .01 & -.05 & -.05 \\
Education & $.26^{* * *}$ & $.23^{* * *}$ & $-.14^{* *}$ & $-.28^{* * *}$ \\
Exposure (annual km) & $.10^{* *}$ & .01 & .01 & .04 \\
Monotonous attention & .01 & -.02 & .03 & .01 \\
Selective attention & $-.20^{* * *}$ & $-.29^{* * *}$ & $.31^{* * *}$ & $.11^{* *}$ \\
Visual pursuit & .05 & .04 & $-.21^{* * *}$ & $-.22^{* * *}$ \\
Eye-hand coordination & -.02 & .01 & .05 & -.01 \\
Reaction time & $-.10^{* *}$ & -.06 & $.14^{* * *}$ & $.15^{* * *}$ \\
Peripheral perception & $.24^{* * *}$ & $.26^{* * *}$ & $-.13^{* *}$ & $-.21^{* * *}$ \\
$\mathrm{R}^{2}$ Change & .11 & .16 & .17 & .14 \\
$\mathrm{R}^{2}$ Total & .32 & .32 & .27 & .39 \\
\hline${ }^{*} \mathrm{p}<.05,{ }^{* *} \mathrm{p}<.01,{ }^{* * *} \mathrm{p}<.001$. & & & &
\end{tabular}

the significant predictor of all outcome variables in the first step. As would be expected, level of exposure to driving (annual $\mathrm{km}$ ) also significantly predicted driver skills. Examination of the betas in the second step (after controlling for demographic variables) indicated that partial correlations were much lower than the correlations suggesting that controlling for demographics lead to a decrease in betas. Selective attention and peripheral perception significantly predicted all of the criterion variables. Selective attention significantly and negatively predicted driving and safety skills and positively predicted violations and errors. This pattern was reversed for the peripheral perception. These findings suggested that high levels of selective attention, in fact, create a proclivity for unsafe driving and aberrant behaviors. Peripheral perception, however, seemed to be critical for safe driving. Visual pursuit was the significant predictor of both driving behaviors, but not the skills. Finally, reaction time weakly but significantly predicted driving skills, violations, and errors.

\section{DISCUSSION}

The results of the current investigation revealed that self-reported driving performance and safety precautions, as well as driving violations and errors, could be systematically predicted by the computerized measures of the cognitive and psychomotor performance. Although the majority of the significant correlations and betas were weak to moderate in magnitude, they were in the expected direction with the exception of selective attention. Unlike other attention variables, selective attention appeared to be persistantly linked with unsafe driving and aberrant behaviors. This could be explained by its association with sensation seeking. Ball and Zuckerman (1992) found that high sensation seekers performed better on a selective attention task than low sensation seekers. Given that sensation seeking is one of the most critical personality variables in 
predicting accident involvement and risk (Jonah, 1997), it may indeed reflect its effect on the risky behaviors. Further studies are needed to better explore the link between selective attention and driving performance. A possible decline in peripheral perception and visual pursuit seemed to be linked with unsafe driving. Future studies should examine if they represent some sort of higher order cognitive and perceptual abilities in driving performance. Results of this study provided initial support for the use of cognitive and psychomotor measures in driver assessment for the young and middle-age population. Our expectation that computerized measures of cognitive and psychomotor abilities would be mainly linked with driving skills and errors, but not safety skills and violations, was not supported. Very high correlations between the skills and behaviors that were measured with the same questionnaire may have resulted in this conclusion. Future studies should examine these possibilities considering the limitations of this study.

\section{ACKNOWLEDGMENTS}

This study was supported by METEKSAN System and Computer Technologies Ltd.

\section{REFERENCES}

Ball, S. A., \& Zuckerman, M. (1992). Sensation Seeking and Selective attention: Focused and divided attention on a dichotic listening task. Journal of Personality and Social Psychology, $5,825-831$.

Brown, I.D., Groeger, J.A. (1988). Risk perception and decision taking during the transition from novice to experienced driver status. Ergonomics 31, 585-598.

Elander, J., West, R., \& French, D. (1993). Behavioral correlates of individual differences in road-traffic crash risk: An examination of methods and findings. Psychological Bulletin, 113, 279-294.

Evans, L., 1991. Traffic Safety and the Driver. New York: Van Nostrand Reinhold.

Hakamies-Blomqvist, L. (1998). Older driver's accident risk: Conceptual and methodological issues. Accident Analyses and Prevention 30, 293-297.

Lajunen, T., \& Summala, H. (1995). Driving experience, personality, and skill and safety motive in drivers' self-assessments. Personality and Individual Differences, 3, 307-318.

Jonah, B. A. (1997). Sensation seeking and risky driving: A review and synthesis of the literature. Accident Analysis and Prevention, 29, 651-665.

Marottoli, R. A., \& Richardson, E. D. (1998). Confidence in, and self-rating of, driving ability among older drivers. Accident Analyses and Prevention, 30, 331-336.

McGwin, G., \& Brown, D. B. (1999). Characteristics of traffic crashes among young, middleaged, and older drivers. Accident Analysis and Prevention 31, 181-198.

McKnight, A.J., \& McKnight, A.S. (1999). Multivariate analysis of age-related driver ability and performance deficits. Accident Analyses and Prevention, 31, 445 -454.

Parker, D., \& Stradling, S.G. (2001). Influencing Driver Attitudes and Behaviors. DETR, Road Safety Research Report No. 17. at http://www. dtlr.gov.uk.

Reason, J., Manstead, A., Stradling, S., Baxter, J., \& Campbell, K. (1990). Errors and violations on the roads. Ergonomics, 33, 1315-1332. 
Sümer, N. (2003). Personality and Behavioral predictors of traffic accidents: Testing a contextual mediated model. Accident Analysis and Prevention, 35 (6), 949-964.

Sumer, N., Ayvasik, B., \& Er, N. (2002). Trafikent Driver Psycho-Technical Driver Assessment System. User Manuel. www.trafikent.com.

SWEROAD Report. (1999). National Road Safety Report and Final Report. General Directorate of Police, Ankara, Turkey. 